\section{Wo sitzt der Schmerz?}

\begin{abstract}
Moderne bildgebende Verfahren machen mit präzisen Darstellungen einzelner Hirnareale Hoffnung, dem genauen Sitz des Schmerzes im Hirn näher zu kommen. Doch offenbar sind verschiedene Schmerzaspekte in unterschiedlichen Regionen repräsentiert.
\end{abstract}

D ie Anwendung bildgebender Verfahren wie die funktionelle Magnetresonanztomografie (fMRT) hat in den letzten Jahren auch in der Schmerzforschung stark zugenommen. Doch Schmerz ist eine multidimensionale Größe mit sensorisch-diskriminativen, affektiv-emotionalen, motorischen, autonomen und kognitiv/attentionalen Komponenten, betonte die Neurologin Professor Ulrike Bingel von der Universität Essen auf dem diesjährigen deutschen Schmerzkongress in Mannheim.

Auf der Suche nach einer spezifischen „Neural Signature of Pain“ (NSP) werden Probanden nach einer experimentellen Stimulation auf die fokale Aktivierung bestimmter Hirnareale hin untersucht. Als Trick dienen dabei parametrische Designs, bei denen mit unterschiedlichen Reizintensitäten getestet wird, ob und wie ein Reiz wahrgenommen wird und ob zugleich eine Stimulus-Intensitätskodierung vorhanden ist. Doch die vermeintliche Identifikation entsprechender Areale ist fehlerbehaftet, so Bingel. Als Beispiel für Fehlinterpretationen sieht die Neurologin etwa Studien, in de-

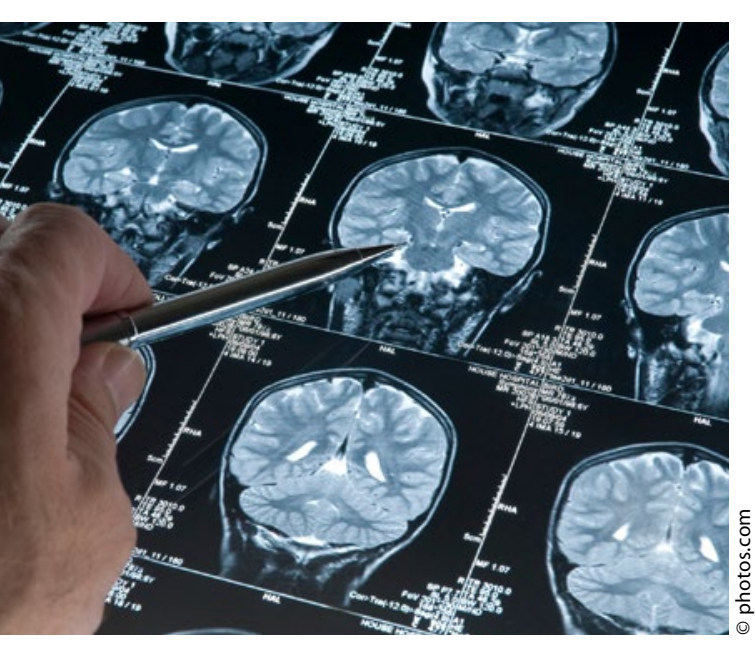

Bildgebende Verfahren können womöglich auch helfen den Ort des Schmerzes zu lokalisieren. nen experimentell erzeugte Situationen sozialer Zurückweisung in Probanden identische Hirnareale aktivierten wie physische Schmerzen.

\section{Dynamisches Netzwerk}

Daraus eine „reverse Inferenz“ abzuleiten, also von reaktiven Hirnarealen auf tatsächlich empfundenen Schmerz zuschließen, „ist nicht zulässig“, erklärte sie. „Es gibt kein Areal im Gehirn, das exklusiv und selektiv nur schmerzhafte Reize verarbeitet." Gewisse Hinweise für ein solches schmerzspezifisches Hirnareal bestehen lediglich für die hintere Insel, die zumindest gehäuft auf Schmerzsignale reagiert [Mazzola et al. Brain. 2012; 135: 631-40]. Generell scheint die individuelle Schmerzerfahrung jedoch in einem dynamischen Netzwerk von Hirnarealen repräsentiert, die auch unterschiedlich miteinander interagieren. „Univariate klassische Mapping-Studien bringen uns dabei nicht weiter, zu sagen, ob jemand Schmerz oder keinen Schmerz oder viel oder wenig Schmerz hat", betonte Bingel.

Ein neuer Trend in der Bildgebung sind multivariate Ansätze mit Bezeichnungen wie „machine learning“, „pattern recognition“ oder „brain reading“. Dabei soll, im Gegensatz zum klassischen Ansatz - bei dem Perzeption die Resultate der Bildgebung mit einzelnen Voxeln erklären soll - die Bildgebung vieler Voxel des Gehirns das Verhalten oder die Perzeption des Probanden erklären. Basierend auf einem Datensatz von Hirnbildern, bei denen Vorhandensein und Intensität des Schmerzes beim Probanden bekannt sind, wird ein Algorithmus formuliert um zwischen verschiedenen Bedingungen $\mathrm{zu}$ unterscheiden. Im Idealfall lässt sich damit die Frage beantworten, ob und wie stark der Schmerz vorhanden ist. Auch der Beitrag einzelner Hirnareale an der Beantwortung dieser Frage lässt sich damit ermitteln. In einer Schlüsselstudie hierzu wurde eine NSP identifiziert, die sich offenbar zur Quantifizierung von experimentell erzeugten thermischen Schmerzen eignet [Wager TD et al. N Engl J Med. 2013; 368: 138897]. Aber schon für eine experimentelle Selbstregulation (hoch/herunter) der Schmerzintensität durch die Probanden ließ sich keine Repräsentation in dieser NSP nachweisen [Woo et al. PLoS Biol. 2015;13:e1002036].

\section{Noch keine klinische Messung}

Dennoch werden die Techniken funktioneller Bildgebung, auch für die Entwicklung zentral wirksamer Schmerzmedikamente bei akuten Schmerzen, zunehmend explorieren. Bislang weitgehend unerforscht sind jedoch noch chronische Schmerzen, so Bingel. Das liege auch daran, dass im Gegensatz zur subakuten Schmerzphase, in der noch klassische Schmerz-assoziierte Netzwerke wie die Insel oder das mittlere Cingulum aktiv sind, sich die zentrale Repräsentation des Schmerzes im Laufe der Chronifizierung in ganz andere Areale verschiebt [Hashimi et al. Brain 2013; 136: 2751-68]. Mit Masken, die eigentlich für die Erfassung emotionaler Prozesse entwickelt wurden, kann dann chronischer Schmerz viel besser erklärt werden als akuter Schmerz. In einer ersten Studie mit diesem Ansatz gelang es bislang allerdings lediglich, zwischen Fibromyalgiepatienten und gesunden Kontrollen zu unterscheiden. Bingels Fazit: Bildgebende Verfahren sind zwar hilfreiche Surrogatmarker zur Darstellung experimenteller Schmerzen im Gehirn, eignen sich bislang aber noch nicht für die Diagnostik und Messung klinischer Schmerzen, zumal es nicht nur eine universelle NPS gibt. Multivariate Ansätze sind noch nicht praxistauglich, aber vielversprechend. Für die genauere Verortung chronischer Schmerzen sind vermutlich weitere, neue Methoden erforderlich. Nicht vergessen werden sollten dabei jedoch die enormen ethischen und juristischen Implikationen dieser neuen Techniken, mahnte die Neurologin.

\section{Dr. Andreas Häckel}

"Schmerz oder kein Schmerz - das ist hier die Frage." Möglichkeiten und Grenzen der Erfassung von akuten und chronischen Schmerzen. Deutscher Schmerzkongress, Mannheim, 22.10.2016 